Accepted for publication in the Astrophysical Journal 10 March 2006, v 639, 2 issue

\title{
The Observed Galactic Annihilation Line: Possible Signature of Accreting Small Mass Black Holes in the Galactic Center
}

\author{
Lev Titarchuk ${ }^{1,3}$ and Pascal Chardonnet ${ }^{2}$
}

\begin{abstract}
Various balloon and satellite observatories have revealed what appears to be an extended source of $0.511 \mathrm{MeV}$ annihilation radiation with flux of $\sim 10^{-3}$ photons $\mathrm{cm}^{-2} \mathrm{~s}^{-1}$ centered on the Galactic Center. Positrons from radioactive products of stellar explosions can account for a significant fraction of the emission. We discuss an additional source for this emission: namely $\mathrm{e}^{+} \mathrm{e}^{-}$pairs produced when X-rays generated from the $\sim 2.6 \times 10^{6}$ solar mass Galactic Center Black Hole interact with $\sim 10 \mathrm{MeV}$ temperature blackbody emission from $10^{17} \mathrm{~g}$ black holes within $10^{14-15} \mathrm{~cm}$ of the center. The number of such Small Mass Black Holes (SMMBHs) can account for the production of the $10^{42} \mathrm{e}^{+} \mathrm{s}^{-1}$ that produces the observed annihilation in the inner Galaxy when transport effects are taken into account. We consider the possibility for confirming the presence of these SMMBHs in the Galactic Center region with future generations of gamma-ray instruments if a blackbody like emission of $\sim 10 \mathrm{MeV}$ temperature would be detected by them. Small Mass Black Hole can be a potential candidate for dark (invisible) matter halo.
\end{abstract}

Subject headings: accretion, accretion disks-black hole physics- radiation mechanisms: nonthermal - physical data and processes-Galaxy:center

\footnotetext{
${ }^{1}$ George Mason University/Center for Earth Observing and Space Research, Fairfax, VA 22030; and US Naval Research Laboratory, Code 7655, Washington, DC 20375-5352; ltitarchuk@ssd5.nrl.navy.mil

${ }^{2}$ Université de Savoie/ LAPTH /LAPP, 9 Chemin de; Bellevue, BP110 74941 Annecy-le-Vieux Cedex FRANCE; chardonnet@lapp.in2p3.fr

${ }^{3}$ NASA/ Goddard Space Flight Center/USRA, code 660, Greenbelt MD 20771; lev@milkyway.gsfc.nasa.gov
} 


\section{Introduction}

The Galactic Center (GC) of our Galaxy was detected in 1974 as a strong radio source called Sgr $\mathrm{A}^{*}$ and is the site where a supermassive black hole with $2.6 \times 10^{6} \mathrm{M}_{\odot}$ is present (e.g. Melia and Falcke 2001). Morever the total mass of stars enclosing the inner 20 pc of the Galaxy contains $\sim 10^{8} \mathrm{M}_{\odot}$, or $\sim 0.1 \%$ of the Galactic stellar mass (Baganoff et al. 2003). Therefore, the X-ray observations of the Galactic Center can potentially reveal accreting black holes and neutron stars. During five years of Chandra observations, Baganoff et al. identified seven X-ray transients located within $20 \mathrm{pc}$ of Sgr A*. Remarkably four of these transients lie within only $1 \mathrm{pc}$ of Sgr A* (Muno et al. 2005). This implies that, compared to the numbers of similar systems located between 1 and $20 \mathrm{pc}$, transients are over-abundant by a factor of $\sim 20$ per unit stellar mass within 1 pc of Sgr $A^{*}$.

Studies of the GC are also a subject of intense activity due to the possible presence of a dark matter cusp. Numerous space missions have observed the center of our Galaxy in radio, infrared, $X$ and $\gamma$ rays. Thanks to these measurements we have a more precise picture of this region. There is a strong activity both in $\mathrm{X}$ and $\gamma$ rays near the center of our Galaxy. A recent HESS (High Energy Stereoscopic System) experiment reported a detection of very high energy gamma rays from the Galactic center within $1^{\prime}$ of $\mathrm{Sgr} \mathrm{A}^{*}$. The flux above $165 \mathrm{GeV}$ is order of $1.8 \times 10^{-7} \mathrm{~m}^{-2} \mathrm{~s}^{-1}$ and consistent with a luminosity of $10^{35} \mathrm{ergs} \mathrm{s}^{-1}$ (Aharonian, et al. 2004).

The most intense galactic gamma-ray line is $511 \mathrm{keV}$ annihilation line. Balloon-flight observations first detected the annihilation line from the Galactic Center region (Johnson, Harnden \& Haymes 1972; see also Johnson \& Haymes, 1973). Since that time the inner Galaxy has been the object of intense observations from space missions and balloon experiments (Leventhal, MacCallum \& Stang 1978; Purcell et al. 1993; Ramaty, Skibo \& Lingenfelter 1994; Purcell et al. 1997; and Cheng et al. 1997; Share et al. 1990; Teegarden et al. 1996; Gehrels et al. 1991; Leventhal et al. 1993; Niel et al. 1990; Chapuis et al. 1991).

Recently the new measurements of the $511 \mathrm{keV}$ line emission from the Galactic Center region have been performed with spectrometer SPI on the space observatory INTEGRAL (Jean et al. 2003). The INTEGRAL observation is in a good agreement with the previous measurements and is well fitted by a gaussian with the full width at half maximum of $\sim$ 10 degrees, with a $2 \sigma$ confidence interval of 6-18 degrees. The INTEGRAL measurement providing the line width of $\sim 2.4 \mathrm{keV}$ exlcudes velocity $v$ of the interstellar medium (ISM) greater than $\sim 800 \mathrm{~km} \mathrm{~s}^{-1}$, i.e. $\beta=v / c \leq 2 \times 10^{-3}$ (Churazov et al. 2005). The source line flux is : $9.9 \times 10^{-4} \mathrm{ph} \mathrm{cm}^{-2} \mathrm{~s}^{-1}$, for which the corresponding luminosity of the line emission is about $6 \times 10^{36}$ ergs $\mathrm{s}^{-1}$ for the distance of about $8 \mathrm{kpc}$. Then the positron sources are produced at a rate given by: $\sim 10^{42} d_{8}^{2} \mathrm{~s}^{-1}$, where $d_{8}$ is the distance expressed in unit of 8 
kpc. In our calculations (see $\S 4$ ) we use this value of the pair flux $10^{42} \mathrm{~s}^{-1}$ inferred from the INTEGRAL observations.

Several possible sources of the observed line related to the positron sources have been proposed. Most of these propose that positrons come from radioactive elements ejected by stellar explosions and massive stars (Matz et al. 1988; Kurfess et al. 1992, Diehl et al. 1995; Iyudin et al. 1994; Dermer \& Skibo 1997). Boehm, Fayet \& Silk (2004) offer an alternative explanation that the observed annihilation line could be related to the annihilation of dark light matter particles into $e^{+} e^{-}$. Guessoum, Jean \& Gillard (2005) review the main processes that lead to the positron production. They are: (i) the $\beta^{+}$decay of radioactive nuclei which is widespread in the explosive and/or hydrostatic nucleosynthesis enviroments of novae, supernovae, Wolf-Rayet, and Asymptotic Giant Branch stars, (ii) $\pi^{+}$decay into $\mu^{+}$and positrons in collisions of highly energetic $(E>200 \mathrm{MeV})$ cosmic rays with interstellar material, (iii) pair production by interaction of an electron with a strong magnetic field and (iv) photon-photon pair production of high energy photons near luminous compact objects, black holes, active nuclei (AGN) etc. The latter origin is consistent with production from interaction of X-ray and $\gamma-$ emission in the very compact shell near Galactic Center as we discuss in this paper.

X-ray observations of the Galactic Center have been made with several instruments (Watson et al. 1981; Skinner et al. 1987; Kawai et al. 1988; Takeshima et al. 1991; Sunyaev et al. 1993; Markevitch et al. 1993; Pavlinsky et al. 1993; Preddehl, \& Trumper 1994). Highsensitivity imaging observations with the Einstein Observatory (Watson et al.) and ROSAT (Preddehl \& Trumper) resolved several discrete sources within $10^{\prime}$ of Galactic Center. One of the ROSAT sources, RXJ 1745.6-2900, is within 10" coincident of the nonthermal radio source Sgr A *, which is believed to be the dynamical center of our Galaxy.

The Japanese satellite GINGA detected $\mathrm{K} \alpha$ lines from highly ionized iron, suggesting a large amount of hot plasma strongly concentrated towards the Galactic Center (Koyama et al. 1989; Yamauchi et al. 1990). The ASCA X-ray image (Tanaka et al. 1995) has revealed extended diffuse emission over the Galactic Center region. The observed spectrum shows the $\mathrm{K}_{\alpha}$ lines from highly-ionized ions of silicon, sulphur, argon, calcium, in addition to high-ionization iron lines. This gives firm evidence for the presence of high-temperature $(k T \approx 10 \mathrm{keV})$ plasma. A large energy input of $\sim 10^{41-42} \mathrm{ergs} \mathrm{s}^{-1}$ is required to produce the observed plasma. Koyama et al. (1996) argue that the source of this energy resides at the Galactic Center. The luminosity required to account for the $6.6 \mathrm{keV}$ line from Sgr B2 (the prominent molecular cloud) is $2 \times 10^{39} \mathrm{ergs}^{-1}$ (in 2-10 keV band). While the Sgr A region has an X-ray luminosity of $\sim 10^{36} \mathrm{ergs} \mathrm{s}^{-1}$ at present, it could have been much more luminous $\sim 300 \mathrm{yr}$ ago. The distinctly bright region inside the Sgr A shell is probably due 
to this recent activity and the $\mathrm{X}$-ray luminosity may have been as high as several $\times 10^{39}$ ergs $\mathrm{s}^{-1}$ at early times. Koyama et al. conclude that the ASCA results suggest the presence of an active galactic nucleus and a large mass concentration at the Galactic Center with transient activities due to sporadic mass accretion rate. Muno et al. (2004a) also presented the study of the spectrum of diffuse emission in $17^{\prime} \times 17^{\prime}$ field around $\mathrm{Sgr} \mathrm{A}^{*}$ during $6 \times 10^{5}$ $s$ of Chandra observations. They confirmed and extended the results of the previous ASCA study. In particular, they showed that the continuum and line spectra are consistent with an origin in a two-temperature plasma: As a cooler, $k T \approx 0.8 \mathrm{keV}$ plasma with varying surface brightness, and a hotter, $k T \approx 8 \mathrm{keV}$ component that is more spatially uniform.

Recently Revnivtsev et al. (2004) have published data on fluorescence in giant molecular cloud Sgr B2's (IGR J17475-2822). They have constructed the broad band (3-200 keV) spectrum from the ASCA-GRANAT-INTEGRAL data. It appears that the X-ray irradiating spectrum of the cloud is, in fact, not free-free, (as early proposed above) but it is rather quite hard, extending to over $100 \mathrm{keV}$ with a power law with photon index 1.8. Revnivtsev et al. concluded that 300-400 years ago Sgr $A^{*}$ was a low luminosity (of $\sim 2 \times 10^{39} \mathrm{ergs} \mathrm{s}^{-1}$ at 2-200 keV) AGN with characteristic hard X-ray Comptonization spectrum with photon index of about 1.8 and plasma temperature of about $50 \mathrm{keV}$.

Gamma-ray emission between $1 \mathrm{MeV}$ and $30 \mathrm{MeV}$ and higher has been measured by COMPTEL and EGRET respectively within a few degrees of the GC cusp. Using the COMPTEL (Strong et al. 1998, 1999, 2004, 2006) and EGRET data (Hunter et al. 1997) one can calculate that the luminosity in the $1-100 \mathrm{MeV}$ energy band is about $4 \times 10^{38}$ ergs $\mathrm{s}^{-1}$ assuming that the distance to the Galactic Center is about $8 \mathrm{kpc}$. In the energy band between 1 and $30 \mathrm{MeV}$ the COMPTEL spectrum presented in units of $E^{2}$ photon intensity $\mathrm{cm}^{-2} \mathrm{sr}^{-1} \mathrm{~s}^{-1} \mathrm{MeV}$ is quite flat (taking into account the error bars). The representative value of the flux in this energy band is about $2 \times 10^{-2} \mathrm{~cm}^{-2} \mathrm{sr}^{-1} \mathrm{~s}^{-1} \mathrm{MeV}$.

In this Paper, we study pair creation due to X-ray and $\gamma$ - photon interactions inside the compact Galactic Center shell (the SGR A shell) and we discuss the possibility of annihilation line formation when these pairs propagate through the Galaxy from the Galactic Center region. In $\S 2$ we present a pair creation model. In $\S 3$ and $\S 4$ we compare our theoretical predictions for the pair and annihilation photon production with observations. In $\S 3$ we determine the size of the pair production area using the observable fluxes in X-ray and $\gamma$-ray energy ranges. In $\S 4$ we investigate the outward propagation of positrons from the Galactic Center region. In $\S 5$ we present arguments for the origin of the $\sim 10 \mathrm{MeV}$ emission near Galactic Center. We discuss our results in $\S 6$ and we make our final conclusions in $\S 7$. 


\section{Pair creation model}

In Figure 1 we present our model for the illumination of the 1-100 $\mathrm{MeV} \gamma$-ray point like sources by X-ray radiation of the central black hole. We assume that the photon density of $\mathrm{X}$-ray photons emitted by the inner shell near the central $\mathrm{BH}$ within $10^{3} R_{\mathrm{S}}$ (Schwarzschild radii) is described by the following distribution over radius $r$

$$
n_{X}=\frac{\mathcal{L}_{X}}{4 \pi r^{2} E_{X} c}
$$

where $\mathcal{L}_{X}$ is X-ray luminosity of the central source per keV (X-ray energy spectrum), $E_{X}$ is the energy of $\mathrm{X}$-ray photon and $c$ is the speed of light. Also we consider that the spherical shell between the inner radius $R_{c}$ and $R\left(R_{c} \leq r \leq R\right.$ and $\left.R \gg R_{c}\right)$ is uniformly filled by $\gamma$-radiation sources where photon density production $P_{\gamma}$ is

$$
P_{\gamma}=\frac{\mathcal{L}_{\gamma}}{E_{\gamma} 4 \pi R^{3} / 3}
$$

where $\mathcal{L}_{\gamma}$ is the $\gamma$-radiation luminosity of the spherical shell per keV ( $\gamma$-ray energy spectrum) and $E_{\gamma}$ is the energy of the $\gamma$-ray photon. Then the pair production by the photons (for which the pair production condition $E_{X} E_{\gamma}>\left(m_{e} c^{2}\right)^{2}$ is satisfied) is

$$
P_{e^{-} e^{+}}^{X, \gamma}=4 \pi \int_{R_{c}}^{R} r^{2} P_{\gamma} d r \int_{\Omega} T_{X, \gamma}(r, \varphi, \theta) d \Omega
$$

where $T_{X, \gamma}(r, \varphi, \theta)$ is the optical path for $\gamma$-ray photon propagating in the X-ray background and incoming at radius $r$ at given direction $\Omega$. The multiple integral in equation (3) can be calculated analytically. In fact,

$$
\begin{gathered}
\int_{\Omega} T_{X, \gamma}(r, \varphi, \theta) d \Omega=\left\{2 \pi \sigma_{X, \gamma}\right\} \times \\
\left\{\int_{0}^{1} d \mu\left[\int_{r\left(1-\mu^{2}\right)^{1 / 2}}^{\infty} n\left(r^{\prime}\right) d s+\int_{r\left(1-\mu^{2}\right)^{1 / 2}}^{r} n\left(r^{\prime}\right) d s\right]+\int_{-1}^{0} d \mu \int_{r}^{\infty} n\left(r^{\prime}\right) d s\right\}
\end{gathered}
$$

where

$$
n\left(r^{\prime}\right) d s=\frac{n_{0} r^{\prime} d r^{\prime}}{r^{\prime 2}\left[r^{\prime 2}-r^{2}\left(1-\mu^{2}\right)\right]^{1 / 2}}
$$

and $n_{0}=\mathcal{L}_{X} / 4 \pi E_{X} c$; where $\sigma_{X, \gamma}\left(E_{X}, E_{\gamma}\right)$ is a pair creation cross section given by (Dirac, 1930, Heitler, 1954)

$$
\sigma_{X, \gamma}\left(E_{X}, E_{\gamma}\right)=\frac{\pi \mathcal{R}_{0}^{2}}{2}\left(1-b^{2}\right)\left[2 b\left(b^{2}-2\right)+\left(3-b^{4}\right) \ln \left(\frac{1+b}{1-b}\right)\right]
$$


and $b=\left[1-\left(m_{e} c^{2}\right)^{2} / E_{X} E_{\gamma}\right]^{1 / 2}$, and where the classical electron radius $\mathcal{R}_{0}=e^{2} / m_{e} c^{2}=$ $2.818 \times 10^{-13} \mathrm{~cm}$.

The analytical integration of $\int n\left(r^{\prime}\right) d s$

$$
\int n\left(r^{\prime}\right) d s=n_{0} \int \frac{d r^{\prime}}{r^{\prime} \sqrt{r^{\prime 2}-r^{2}\left(1-\mu^{2}\right)}}=\frac{n_{0}}{r \sqrt{1-\mu^{2}}} \arccos \frac{r \sqrt{1-\mu^{2}}}{r^{\prime}}+C
$$

allows us to calculate the second integral of $\int n\left(r^{\prime}\right) d s$ over $\mu$ :

$$
\int_{\Omega} T_{X, \gamma}(r, \varphi, \theta) d \Omega=2 \pi \sigma_{X, \gamma} \frac{\mathcal{L}_{X}}{4 \pi E_{X} c} \frac{3 \pi^{2}}{4 r}
$$

Finally, the expression for the Green's function $P_{e^{-} e^{+}}^{X, \gamma}$ can be written as

$$
P_{e^{-} e^{+}}^{X, \gamma}=\frac{9 \pi^{2}}{16 c R} \frac{\sigma_{X, \gamma} \mathcal{L}_{X} \mathcal{L}_{\gamma}}{E_{X} E_{\gamma}}
$$

In order to obtain the total pair production rate $P_{e^{-} e^{+}}$one should integrate $P_{e^{-} e^{+}}^{X, \gamma}$ over X-ray and $\gamma$ - energies, namely

$$
P_{e^{-} e^{+}}=\frac{9 \pi^{2}}{16 c R} \int_{0}^{\infty} d E_{X} \int_{\left(m_{e} c^{2}\right)^{2} / E_{X}}^{\infty} \frac{\sigma_{X, \gamma} \mathcal{L}_{X} \mathcal{L}_{\gamma}}{E_{X} E_{\gamma}} d E_{\gamma}
$$

\section{Results of calculations}

To compute the positron flux one needs the $\mathrm{X}$ and $\gamma$ ray energy spectra, $\mathcal{L}_{X}$ and $\mathcal{L}_{\gamma}$ respectively. We will use for the total $X$-ray and $\gamma$ luminosities of the inner $\mathrm{GC}$ region, $L_{X} \sim 2 \times 10^{39} \mathrm{ergs}^{-1}$ (Revnivtsev et al. 2004) and $L_{\gamma} \sim 4 \times 10^{38} \mathrm{ergs} \mathrm{s}^{-1}$ (e.g. Strong et al. 1999) respectively (see a review of the observations in the Introduction).

We assume that the $\gamma$ - radiation emanates from a optically thick medium (as an accretion disk around the compact object) and consequently the emergent spectrum has a blackbody like shape that color temperature $T_{\gamma}=10 \mathrm{MeV}$ (see Fig. 1). Then for a normalization constant $C_{\gamma}$ we have an equation as follows

$$
L_{\gamma}=\int_{0}^{\infty} \mathcal{L}_{\gamma}\left(E_{\gamma}\right) d E_{\gamma}=C_{\gamma} \int_{0}^{\infty} \frac{y^{3}}{\exp (y)-1} d y
$$

where $y=E_{\gamma} / k T_{\gamma}$. For the X-ray photons, we assume a power law with an exponential cutoff

$$
L_{X}=\int_{0}^{\infty} \mathcal{L}_{\mathcal{X}}\left(E_{X}\right) d E_{X}=C_{X} \int_{0}^{\infty} x^{-\alpha} \exp (-x) d x
$$


where $x=E_{X} / k T_{X}, k T_{X}=100 \mathrm{keV}$, the spectral index $\alpha=\Gamma-1=0.8$ and $C_{X}$ is the normalization constant. From the previous section we saw that the process of pair creation is driven by the cross section given by equation (6). The energy dependence, represented by the parameter $b$ can be expressed using the dimesionless energies $x=E_{X} / k T_{X}$ and $y=E_{\gamma} / k T_{\gamma}$, namely $b=\left[1-\left(m_{e} c^{2}\right)^{2} / E_{X} E_{\gamma}\right]^{1 / 2}=[1-3.26 / x y]^{1 / 2}$. Then the formula for the pair flux

$$
P_{e^{-} e^{+}}=\frac{9 \pi^{2}}{16 c R} \int_{0}^{\infty} d E_{X} \int_{\left(m_{e} c^{2}\right)^{2} / E_{X}}^{\infty} \frac{\sigma_{X, \gamma}\left(E_{X}, E_{\gamma}\right) \mathcal{L}_{\gamma}\left(E_{\gamma}\right) \mathcal{L}_{\mathcal{X}}\left(E_{X}\right)}{E_{X} E_{\gamma}} d E_{\gamma}
$$

can be modified using the variables $x$ and $y$ as follows

$$
P_{e^{-} e^{+}}=\frac{9 \pi^{2}}{16 c R} \int_{0}^{\infty} d x \int_{3.26 / x}^{\infty} \frac{\sigma_{E_{X}, E_{\gamma}}(x, y) \mathcal{L}_{\gamma}(y) \mathcal{L}_{\mathcal{X}}(x)}{x y} d y
$$

We compute this double integral and obtain the following result:

$$
P_{e^{-} e^{+}}=\frac{3.2 \times 10^{56}}{R} \mathrm{~cm}^{-1} \mathrm{~s}^{-1}
$$

To reproduce the value of $10^{42} \mathrm{~S}^{-1}$ for the positrons rate, one needs a value of the radius $R$, given by:

$$
R=\frac{3.2 \times 10^{56}}{P_{e^{-} e^{+}}}=\frac{3.2 \times 10^{56}}{10^{42}}=3.2 \times 10^{14} \mathrm{~cm}
$$

It is worth noting that the intrinsic pair flux $\sim 10^{36} \mathrm{~s}^{-1}$, formed as a result of $\gamma+$ $\gamma$ interaction (to calculate this flux one should replace $\mathcal{L}_{\mathcal{X}}$ by $\mathcal{L}_{\gamma}$ in formula 14) in this $\gamma$-emission region of size $3.2 \times 10^{14} \mathrm{~cm}$, is six orders of magnitude less than the pair flux $\sim 10^{42} \mathrm{~s}^{-1}$ required to reproduce the observed annihilation line strength.

\section{Outward propagation of positrons from the Galactic Center region}

A positron, $e^{+}$may collide with an electron $e^{-}$, to produce two gamma ray photons according to the reaction $e^{+}+e^{-} \rightarrow \gamma+\gamma$. Before decaying, the positron in free space can interact with an electron and to form positronium, a bound state with lifetime of order of $10^{-10} \mathrm{~s}$. The particles annihilate into two or three photons depending whether it is formed in the singlet or triplet state. In our calculations we assume that most of the positrons are thermalized in the surrounding thermal plasma and thus we can consider the annihilation of thermal positrons at a temperature of order $10^{7} \mathrm{~K}$, i.e. $\beta=\langle v\rangle / c \sim 10^{-3}$, where $\langle v\rangle$ is the thermal velocity of electrons. In fact, the recent INTEGRAL observations of $511 \mathrm{keV}$

annihilation line (Churazov et al. 2005) exclude velocities of the interstellar medium greater 
than $10^{-3} c$. For $\beta \ll 1$, the cross-section $\sigma$, for positron annihilation with a free electron at rest is given by Dirac (1930)

$$
\sigma \approx \pi r_{0}^{2} / \beta
$$

The problem of the positron thermalization and annihilation photon production in the interstellar medium has been recently studied in details by Guessoum, Jean, \& Gillard (2005) (see for a review of this subject). They reexamine in detail the various processes for positron annihilation using most recent interaction cross section with atomic and molecular hydrogen, as well as helium. Putting all the new calculations together, they constructed annihilation spectra of ISM.

The fundamental question is how far the positrons of energy about one MeV can travel from the Galactic central region to be annihilated. In order to answer to this question one has to compare the diffusion (travel) time with the energy loss (thermalization) time taking into account the main energy loss mechanisms. In general terms, the particle diffusion is related to escape of a magnetized turbulent plasma.

Jean et al. (2005) analyze the positron diffusion propagation in the GC region in detail. The quasilinear diffusion coefficient $D_{q l}$ (Melrose 1980) can be expressed as:

$$
D_{q l}=D_{\mathrm{B}}\left(\frac{r_{\mathrm{L}}}{\lambda_{\max }}\right)^{1-\delta} \eta^{-1}
$$

with $D_{\mathrm{B}}=(1 / 3) v r_{\mathrm{L}}$ the Bohm diffusion coefficient, $\lambda_{\max }$ is the maximum scale of the turbulence, $\delta=5 / 3$ for a Kolmogorov turbulent spectrum, $\eta=\delta B^{2} /\left\langle B^{2}\right\rangle$ is relative perturbation of the magnetic field pressure which is often approximated to $1, v$ is the positron velocity and $r_{\mathrm{L}}$ is the Larmor radius (gyroradius)

$$
r_{\mathrm{L}}=\frac{m_{e} c \gamma v}{q B}
$$

$q$ is the positron (electron) charge, $B$ is the magnetic field strength, $\gamma=\left[1-(v / c)^{2}\right]^{-1 / 2}$. The maximum scale $\lambda_{\max }$ was estimated to be $\sim 100 \mathrm{pc}$ from the measurements of ISM turbulence (Armstrong et al. 1995).

Recently the (mean) magnetic field strength in the GC region was measured by La Rosa et al. (2005), hereafter LaR05, to be $\sim 10 \mu \mathrm{G}$. LaR05 have used the Very Large Array in all four configurations to image the $\mathrm{GC}$ region at $74 \mathrm{MHz}$. The resulting image of large scale $\left(6^{\circ} \times 2^{\circ}\right)$ of nonthermal synchrotron emission presented in LaR05 has a resolution of $125^{\prime \prime}$. At distance of $8 \mathrm{kpc}$ the angular scales of $6^{\circ} \times 2^{\circ}$ corresponds to region $840 \mathrm{pc} \times 280 \mathrm{pc}$ and that of $125^{\prime \prime}$ corresponds to $0.09 \mathrm{pc}$. It has be noted that in the past several authors (see Morris \& Serabyn et al. 1996 and references therein) estimated magnetic fields values 
of $\sim m \mathrm{G}$ in the GC region. This is 2 orders of magnitude larger than the value $(\sim 10 \mu \mathrm{G})$ obtained by LaR05.

Thus for the mean $\mathrm{B}$ of $10 \mu \mathrm{G}$ and for $1 \mathrm{MeV}$ positrons $D_{q l} \sim 2.6 \times 10^{26} \mathrm{~cm}^{2} \mathrm{~s}^{-1}$. The low limit time scale for $1 \mathrm{MeV}$ positrons to thermalize in the $B \sim 10 \mu \mathrm{G}$ and $\sim 1 \mathrm{~cm}^{-3}$ density region due to ionization, bremsstrahlung, synchrotron and other processes is $\tau_{\sim}^{>} 10^{5}$ years. Then the distance travelled by diffusion in a time of $\tau_{d} \sim 10^{7}$ years (slowing down time plus annihilation time in the standard grain model) is

$$
d_{q l}=\sqrt{D_{q l} \tau_{d}}=3 \mathrm{kpc}
$$

which is much greater than typical half-size of the hot GC region. The volume filling factor of the hot $\mathrm{GC}$ region 0.72 is highest among all phases of the Galactic bulge (see more details of these estimates in Jean et al. 2005). Using the spectral data and positron propagation analysis Jean et al. (2005) come to conclusion that the sources of annihilation emission are diffusively distributed over the Galaxy. They also explain that the lack of annihilation emission from the GC hot gas is due to its low density, which allows positrons to escape this phase.

It is worth noting Liu, Petrosian \& Melia (2004) find that an outflow of low energy electrons of order $1 \mathrm{MeV}$ are distributed over a spatially larger scale than that of the $\mathrm{BH}$ inner region (see more discussion of their work in section 6.2). Namely, the escape time of these electrons is more than a factor of 2 shorter than the time scales for acceleration and losses.

On the hand one can argue that the positrons can be diffusely trapped, thermalized and annihilated in a region of size $L$ of order a few parsec where the magnetic field can be much higher than $\sim 10 \mu \mathrm{G}$. Melia \& Falcke (2001) suggest that out to $\sim 2-3$ pc (essentially inside the cavity surrounded by the circumnuclear disk), the field is turbulent (matching the turbulent plasma generated by wind-wind interactions from the Wolf-Rayet and O/B stars in this region), and can be as high as $\sim 0.2-1 \mathrm{mG}$.

The problem with such fields is that the gyroradius of the electrons and positrons produced in gamma-gamma interactions, will be far smaller than the region over which the positrons are believed to be annihilating. Consequently, the pairs should be radiating profusely via synchrotron, bremsstrahlung, and inverse Compton.

Another estimate of the magnetic field comes from equipartition with the photon density. The photon energy density in this region is about $1 \mathrm{eV} \mathrm{cm}^{-3}$. To obtain this number we assume that the bolometric luminosity of the source in the Galactic Center is of order $10^{38}$ erg s $^{-1}$ (see e.g. Narayan et al. 1998). Thus the derived magnetic strength $B_{b o l}$ using equipartition between photons and magnetic field energies is about $10 \mu \mathrm{G}$. For such a low 
magnetic field we have already demonstrated that the positrons can escape from the GC region.

Longair (1994) also derived the minimum magnetic energy requirement for a given luminosity $\mathcal{L}_{\nu}$ and photon volume $V$ per unit time (in our case $V=4 \pi L^{2} c$ ). He shows that $B_{\min } \propto$ $\left(\mathcal{L}_{\nu} / V\right)^{2 / 7}$ a value that is higher than that obtained using the relation $\mathcal{L}_{\nu} /\left(4 \pi L^{2} c\right)=B_{b o l}^{2} / 8 \pi$. But the ratio of $B_{\text {min }} / B_{b o l}$ is not large. The indicies of the ratios $\mathcal{L}_{\nu} / V$ and $\mathcal{L}_{\nu} /\left(4 \pi L^{2} c\right)$ are $2 / 7$ to $1 / 2$ respectively. Furthermore Longair found that the magnetic field strength, $B_{m i n}$, occurs when there is near equality of the energies in the particles and magnetic field. LaR05 confirm this conclusion. They show that the mean magnetic field in the GC region $10 \mu \mathrm{G}$ inferred from their observations is consistent with the particle energy density of $1.2 \mathrm{eV}$ found in cosmic-ray data.

The turbulent diffusion time scale $\tau=L^{2} / D_{q l}$ is not only determined by the magnetic field. In fact for $\delta=5 / 3$ the diffusion coefficient $D_{q l}$ is proportional to $B^{-1 / 3}$ (see Eq.18). Even if the magnetic field is about $1 \mathrm{mG}$ the diffusion coefficient $D_{q l}$ decreases by factor 4.6 only, namely $D_{q l} \sim 5.5 \times 10^{25} \mathrm{~cm}^{2} \mathrm{~s}^{-1}$. The related difussion time through this region $\tau$ is about $5 \times 10^{4}$ years which is much less than the thermalization and annihilation time $10^{7}$ years.

\section{Emission from small mass BHs as a source of $\gamma$-radiation}

One of the possible origins for $\mathrm{MeV}$-emission is a disk emission from mass accretion by Small Mass Black Holes (SMMBHs). The BH mass can be evaluated using the color disk temperature $T_{c o l}$. Using formula (5) in Shrader \& Titarchuk (1999), hereafter ShT99, we obtain that a black hole mass in solar units $\left(m=M_{b h} / M_{\odot}\right)$ is:

$$
m=\dot{m} \frac{(1 / 7) 3^{4} T_{h}^{4}}{\left(T_{\max }\right)^{4}\left[(7 / 6)^{2} r_{i n}\right]^{3}}
$$

$\dot{m}=\dot{M} / \dot{M}_{\text {Edd }}$ is the dimensionless mass accretion rate in units of $\dot{M}_{\text {Edd }}=L_{\text {Edd }} / c^{2}$, related to the Eddington luminosty $L_{\mathrm{Edd}}, T_{\max } \approx 1.2 T_{\text {col }}$ is the maximum temperature in the disk in $\mathrm{keV}, T_{h}=T_{c o l} / T_{\text {eff }}$ is a disk color factor and $r_{i n}$ is the inner disk radius calculated in Schwarzchild units. Analyzing quite a few BH sources, ShT99 and Borozdin et al. (1999), hereafter BRT99, established that in the soft state the inner disk radius is very close to the last stable Keplerian orbit, namely $r_{i n} \approx 3$. The disk color factor $T_{h} \approx 2.6$ was calculated by BRT99 using the known contraints on BH mass and the distance to the source for GRO J1655-40. This value of the disk color factor $T_{h} \approx 2.6$ obtained for GRO J1655-40 was recently confirmed for other Galatic black hole candidates sources. Shrader \& Titarchuk 
(2003) have made use of improved Galactic black hole binary dynamical mass determinations to derive, in effect, an empirical calibration of this factor.

In the soft state the mass accretion rate in the disk $\dot{m}=\dot{M} / \dot{M}_{\text {Edd }}$ is on the order of Eddington, i.e $\dot{m} \approx 1$. With assumption regarding $r_{i n}, T_{h}$ and $\dot{m}$ we obtain

$$
m=3.7 \times 10^{-16}(\dot{m} / 1) \frac{(1 / 7) 3^{4}\left(T_{h} / 2.6\right)^{4}}{\left(T_{\max } / 1.2 \cdot 10^{4} \mathrm{keV}\right)^{4}\left[(7 / 6)^{2}\left(r_{i n} / 3\right]^{3}\right.}
$$

The SMMBHs can be exposed through the accretion, if the mass accretion is at the Eddington rate and higher. The Eddington accretion rate by definition is

$$
\dot{M}_{E d d}=L_{E d d} / c^{2}=1.4 \times 10^{17} \mathrm{mg} \mathrm{s}^{-1} \text {. }
$$

Thus for SMMBH of $m=m_{s m m b h}=3.7 \times 10^{-16}$ (the SMMBH mass in grams is $\sim 7 \times 10^{17}$ g) the Eddington rate is about $52 \mathrm{~g} \mathrm{~s}^{-1}$. The gravitation attraction of the black hole in the Galactic center causes a substantial mass accretion in its proximity. This accretion flow passing through SMMBH which are probably distributed uniformly around the central black hole, provides enough material for mass supply of the SMMBH objects.

Sgr $\mathrm{A}^{*}$, the massive $\mathrm{BH}$ in the Galactic Center was recently found to be surrounded by a cluster of young, massive stars. Over the past year, three of these stars named SO-2, SO-16 and SO-19 by Ghez et al (2004) and Schodel et al (2003) were determined to be at $\sim 10^{3}$ Schwarzschild radii from the central black hole. It was also found that these stars can be classified as $\mathrm{O}$ stars in the main sequence (Ghez et al. 2003; Eisenhauer et al. 2003). The winds from these stars have a typical mass loss $\dot{M}_{w} \sim 10^{-6} M_{\odot} \mathrm{yr}^{-1}$ and speed in the range $v_{w}=(1-3) \times 10^{3} \mathrm{~km} \mathrm{~s}^{-1}$ (Puls et al. 1996; Repolust, Puls \& Herrero 2004). Loeb (2004) showed that SO-2, SO-16, SO-19 can supply the required mass flow that fuels the emission from $\operatorname{Sgr} A^{*}$.

Now we can check if this mass accretion rate $\dot{M}_{w} \sim 10^{-6} M_{\odot} \mathrm{yr}^{-1} \sim 10^{20} \mathrm{~g} \mathrm{~s}^{-1}$ can provide the Eddington mass accretion into a SMMBH of $\sim 50 \mathrm{~g} \mathrm{~s}^{-1}$. In order to do this one should calculate the SMMBH luminosity of $m_{s m m b h}=3.7 \times 10^{-16}$ for a given color temperature $T_{\text {col }}=10^{4} \mathrm{keV}$ as follows (BRT99)

$$
L_{s m m b h}=1.4 \times 10^{34}\left(\pi^{5} / 15\right) m^{2} r_{\text {eff }}^{2}\left[T_{\text {col }}(\mathrm{keV}) / T_{h}\right]^{4} \mathrm{ergs} \mathrm{s}^{-1} \approx 2 \times 10^{21} \mathrm{ergs} \mathrm{s}^{-1}
$$

where $r_{\text {eff }} \sim 15$ is the disk effective radius in Schwarzschild units, and the color factor $T_{h} \sim 2.6$ (see BRT99). It is worth noting that we choose the color temperature $T_{\text {col }}=10^{4}$ $\mathrm{keV}$ based on the COMPTEL-EGRET observations (see the Introduction).

Then we can evaluate the number of SMMBHs $N_{s m m b h}$ in the GC region if we assume they are responsible for $\gamma$-ray emission about $4 \times 10^{38}$ ergs $\mathrm{s}^{-1}$, namely $N_{\text {smmbh }}=4 \times$ 
$10^{38} / 2 \times 10^{21}=2 \times 10^{17}$. Note that the total mass of SMMBHs $m_{t o t}=M_{t o t} / M_{\odot}$ inside the region of radius $R \sim 3 \times 10^{14} \mathrm{~cm}$ is $m_{\text {tot }}=m_{s m m b h} N_{s m m b h} \stackrel{\sim}{\sim}$. One can conclude that the mass flow rate $\dot{M}_{s m m b h} N_{s m m b h}=10^{19} \mathrm{~g} \mathrm{~s}^{-1}$ needed to fuel SMMBH in the Galactic Center region within $10^{14} \mathrm{~cm}$ (or within $\sim 10^{3}$ Schwarzschild radii from the central $\mathrm{BH}$ ) is much smaller then the mass flow $10^{20} \mathrm{~g} \mathrm{~s}^{-1}$ supplied by the young massive O-stars in this central region. Consequently, we have shown how the small mass black holes could be become exposed when there is enough material around them.

\section{Discussion}

\subsection{The spectrum and origin of X-ray emission of a $\mathrm{BH}$ in the Galactic Center}

Revnivtsev et al. (2004) claimed that the inferred X-ray spectrum and luminosity of the emission illuminating the molecular cloud Sgr B2's, strongly support the idea that the X-ray emission of Sgr B2 is Compton scattered and reprocessed radiation emitted in the past by the Sgr $\mathrm{A}^{*}$ source. We have calculated the pair creation using the inferred luminosity and the inferred spectrum that is a power-law photon spectrum of index 1.8 with an exponential cutoff at $100 \mathrm{keV}$. In fact, this is a typical spectrum of $\mathrm{BH}$ sources at low/hard state which is presumably a result of Comptonization of the soft (disk) radiation in the hot electrons of temperature $50 \mathrm{keV}$ in Compton cloud around central BH.

It has already been shown that the spectral shape in the low hard state is independent of the luminosity (e.g. Laurent \& Titarchuk 1999, Titarchuk \& Fiorito 2004). We can propose that $\operatorname{Sgr} A^{*}$ is now in a quiescent state, presumably a result of the gravitational energy release in the advection dominated flow (see e.g Narayan \& Yi 1994).

The luminosity of the X-ray spectrum of the X-ray source IGR J17475-2822 has not changed since GRANAT/ART-P and ASCA era, i.e. during last 10 years. The clear match of ASCA/GIS, GRANAT/ART-P and INTEGRAL/IBIS (Fig. 2, Revnivtsev et al. 2004) strongly supports this claim. Thus the proposed incident spectrum emanating from Sgr A* in the past (300 years ago) should not be vary either during the period at least of 10 years. It means that the inferred e+e- rate should be quite stable during the same period. The constraints on the "breathtaking variability on a time scale of only several hundred years" was proposed by Sunyaev et al. (1993). They argue that X-ray echo from Sgr B2 should be delayed by $300-400$ years relative to the direct signal from Sgr $\mathrm{A}^{*}$ due to the light travel time from Sgr $\mathrm{A}^{*}$ to Sgr B2. One can put the important question regarding such a short recurrence time for the spectral transition, as 300-400 years for a black hole of mass $\sim 3 \times 10^{6}$ 
solar masses.

On the other hand one can suggest the more likely scenario for irradiating Sgr B2 was the interaction between the supernova remnant, we now see as Sgr A East and the $50 \mathrm{~km}$ $\mathrm{s}^{-1}$ molecular cloud behind Sgr $\mathrm{A}^{*}$, rather than Sgr $\mathrm{A}^{*}$ itself. The luminosity, time scale, and spectrum all fit the requirements rather well, so the need for a high X-ray activity in Sgr $A^{*}$ can be weaker now. This effect of supenova (SN) ejecta and its interactions with molecular clouds proposed by Bykov $(2002,2003)$ is recently discussed in the literature (see e.g. Muno et al. 2004b, and Park et al. 2004). One can be right that "the luminosity, time scale, and spectrum all fit the requirements rather well". However Park et al. pointed out that although the SN ejecta contribution to the observed neutral Fe line emission appears to be plausible, they noted a caveat, that nonthermal radio emission features have not been reported in the northeast regions of $\operatorname{Sgr} \mathrm{A}^{*}$.

This absence of nonthermal radio features is admittedly a difficult problem in terms of the SNR interpretation. It means that the supernova ejecta model needs the nonthermal distribution of electrons in order to explain the hard X-ray energy spectrum of the Sgr B2 molecular cloud (Revnivtsev et al. 2004). We want to emphasize once again that the spectrum seen from Sgr B2 is a typical spectrum of the low/hard state seen in many BH

sources. It has already been proven long ago (see e.g. Sunyaev \& Titarchuk 1980) that this hard spectrum is a result of thermal Comptonization of soft (disk) photons in the hot Compton cloud surrounding the central part of a $\mathrm{BH}$. Thus one does not need any fine tuning or nonthermal electron distribution to produce such a spectrum, because in the outskirts of the GC BH there are plenty of molecular clouds, and we should see the effect of the reflection of the BH X-ray spectrum from these configurations. Thus the spectra of molecular clouds are naturally formed as a result of reflection of $\mathrm{BH}$ emission from molecular clouds.

\subsection{The site and the origin of the annihilation line}

In connection with the previous points, one can raise a question related to expected rapid variability in the $e+e-$ annihilation line flux for such a compact $\mathrm{BH}$ region. However the observations over several decades seem to not show any variability at all.

It is correct to say that the variability in the $e+e-$ annihilation line flux would be quite high if this line was formed in the compact central region. However, we propose that the pairs are produced in this compact region and only then do they propagate through the Galaxy and the e+e- annihilation line is formed through the Galaxy. Thus variability of the $\mathrm{e}+\mathrm{e}-$ annihilation line flux is related to the scale of a few $\mathrm{kpc}$ and it is not by chance that 
observations over several decades seem to not show any variability at all.

We infer that the $\sim \mathrm{MeV}$ gamma ray region, where the pairs are created due to X-ray illuminations by the central source, is quite compact. Its radius is $\sim 3 \times 10^{14} \mathrm{~cm}$. This region (within $10^{14-15} \mathrm{~cm}$ ), is consistent with the COMPTEL map (Schonfelder et al. 2000, Strong et al. 1998). The full sky intensity map, longtitude and latitude profiles for 1-3, 3-10 and $10-30 \mathrm{MeV}$ show the strong peaks within a few degrees near GC. However, the spatial resolution of the COMPTEL map is not better than one degree and thus the compact region of $\sim 10^{14-15} \mathrm{~cm}$ cannot be resolved in COMPTEL map. It is worth noting that Strong, Moskalenko \& Reimer (2000) argue that there is an unresolved point-source population in the inner Galaxy and that this source is an important contribution to the emission around $10 \mathrm{MeV}$. Furthermore, recently Strong et al. (2005) confirm that their model spectrum compatible with EGRET and Cosmic-ray measurements still lies about a factor 2 below the COMPTEL data, as found previously (Strong et al. 2000). There they propose that a contribution from compact sources is the most likely explanation.

Producing $\sim 10^{42} \mathrm{~s}^{-1}$ pair flux (or more, depending on the duty cycle) has consequences beyond simply calculating the annihilation rate. The region surrounding Sgr A* is magnetized, with magnetic field strength $B$ of order $10 \mathrm{G}$ inside $10^{14} \mathrm{~cm}$. Out to $\sim 2-3 \mathrm{pc}$ the field is turbulent (matching the turbulent plasma generated by wind-wind interactions from the Wolf-Rayet and O/B stars in this region). Melia \& Falcke (2001) have suggested that the magnetic field strength of this region should be in the range of $\sim 0.2-1 \mathrm{mG}$.

In section 4.2 we review the positron propagation in the Galactic Center region and we find the positrons can pass through there. Consequently the sources of annihilation emission are presumably distributed over the Galaxy.

Recently Liu, Petrosian \& Melia (2004), hereafter LPM04, formulated and solved the propagation and acceleration problem in the turbulent magnetized plasma for a given pair flux $Q$, magnetic field strength $B$, plasma interaction time $\tau_{p}$, density $n$, the size of the acceleration site $R$ and particle distribution of the background plasma. They showed that acceleration of electrons by plasma wave turbulence in hot gas in the inner-most part of the black hole reasonably account for Sgr $\mathrm{A}^{*}$ 's millimeter and shorter wavelength emission in the quiescent state and for the infrared and X-ray flares. In their model $\mathrm{A}\left(Q=3.2 \times 10^{42}\right.$ $\mathrm{s}^{-1}, B \sim 9 \mathrm{G}$ ) they found the power carried away by accelerated electrons, with $\gamma>100$, is about $2 \times 10^{37}$ ergs s$^{-1}$; which is more than enough to power observed radio emission, whose liminosity of $10^{34}-10^{35} \mathrm{ergs} \mathrm{s}^{-1}$.

For the pair flux of order $10^{42} \mathrm{~s}^{-1}$, the "collateral" spectrum (and flux) arising from these particles is very consistent with $\mathrm{Sgr} \mathrm{A}^{*}$ 's millimeter and shorter wavelength emission 
in the quiescent state and for the infrared and X-ray flares (LPM04). It is worth noting that the low energy electron flux $E \gtrsim m c^{2}$ calculated by LPM04, is comparable with the injected flux $Q$. It means that a significant fraction of the low enery electrons escapes from this region without any noticeable acceleration. In fact, the escape time of low energy electrons is more than a factor of 2 shorter than the time scales for acceleration and losses. The exact value of the electron flux depends on the model assumptions (see LPM04); and thus these theoretical uncertainties of the flux estimate (within a factor of a few) are unavoidable. The LPM04's model also suggested that an outflow of high energy electrons (Liu \& Melia 2002) are distributed over a spatially larger scale than that of the $\mathrm{BH}$ inner region. Finally the escaping high energy electron gas (cooled through the radiation) could form the annihilation line emission in the large scale of the Galaxy. The LPM04's injected flux $Q \sim 10^{42} \mathrm{~s}^{-1}$, is related to the mass accretion rate of order $10^{-5}$ of the Eddington value $\left(\dot{M}_{\mathrm{Edd}}=L_{\mathrm{Edd}} / c^{2}\right)$. If the pairs are accelerated to $\gamma>100$ then their energy would be enough to power the observed radio emission whose luminosity is about $10^{34}-10^{35} \mathrm{ergs} \mathrm{s}^{-1}$.

It is worth noting that within our model, the same flux of pairs $\left(Q \sim 10^{42} \mathrm{~s}^{-1}\right)$ is generated in the SMMBH site as a result of X-ray illumination emitted by surrounding material. If at the present time the X-ray flux is of order $10^{36} \mathrm{erg} \mathrm{s}^{-1}$, then the pair flux would be $10^{39} \mathrm{~s}^{-1}$.

\subsection{The dark matter problem and its relation to observed annihilation line flux}

Dark matter is a long standing puzzle starting from Le Verrier with his discovery of Neptune in 1846 as a perturbation of Uranus (Le Verrier 1846). Our Galaxy is surrounded by an extended halo of unseen material. So far undetected, that dark matter induces a flat rotation curve in the Galactic plane (van Albada \& Sancisi 1986). Its presence has been noticed since many decades (see Oort 1932). However, its nature is still unknown. The dark matter problem consists simply, in the existence of invisible mass showing its presence by gravitational effects. Now, it is widely accepted that dark matter exists. In fact, there is evidence for dark matter on scales from galaxy to the cluster of galaxies and to the whole Universe itself. Zwicky (1937) measured the dispersion velocity in the Coma cluster and found that the dynamical mass was hundred times more than the luminosity mass.

Moreover, the dark matter seems essential: the growth of structure in the Universe by hierachical merging of the dark matter halo is a master piece of moderm cosmology. Recently, WMAP has established the presence of non baryonic dark matter with the density reported in terms of critical density of order $\Omega_{m}=\rho_{m} / \rho_{c} \simeq 0.3$. This is now the standard value of 
the modern cosmology for dark matter density.

On the galactic scale, one can interprete the rotation curve measurements by the presence of the dark matter halo and then determine the mass density profile of this distribution. The average density is $\rho_{m} \simeq 0.3 \mathrm{GeV} \mathrm{cm}{ }^{-3}$. The core of this halo seems to be extremely packed. In the Galactic Center, numerical simulations of structure formation in the nonlinear regime have shown the presence of a singular power law cusp $\rho(r)=\rho_{0}\left(r / r_{0}\right)^{-\gamma}$, where $0<\gamma_{\sim} 2$ and $\rho_{0}$ is the dark matter density at radius $r_{0} \simeq 2 \mathrm{pc}$ (Gnedin \& Primack 2004). The central density $\rho_{0}$ is not uniquely determined and its value depends on models and it varies from 60 to $600 M_{\odot} \mathrm{pc}^{-3}$. On the other hand up to now there is no identification for it despite the large number of candidates proposed from masses in the range between $10^{-5}$ $\mathrm{eV}$ to $10^{12} M_{\odot}$.

While there is no a priori reason to expect that massive compact halo objects (MACHOs) in the mass range $10^{-15} M_{\odot} \lesssim M<10^{-7} M_{\odot}$ comprise a significant fraction of the density of the Universe, neither is there any definitive argument ruling them out. Nemiroff \& Gould (1995) and Marani et al. (1999) discuss the possibility of identification of SMMBHs by gravitational lensing. The basic idea is to study the deflection of light by measuring the amplification due to gravitational bending of compact objects. For black hole mass in the range $10^{-16} M_{\odot}$ to $10^{-13} M_{\odot}$, the expected image separation is of order of $10^{-15}$ arcseconds and the average time separation between the images is order of $10^{-24} \mathrm{~s}$. They applied this delay as an interference process using gamma ray bursters. On the other hand, the absolute value of the light deflection by small black holes is extremely small $\left(10^{-12} \mathrm{~cm}\right) / R$, where $R$ is the characteristic distance in the Galaxy and therefore not observable. It is easy to show that the optical depth for the photons scattering at small angles by SMMBH is small, $\left(\sim 10^{-6}\right)$ and consequently the light multiple scattering by the small black holes is also ruled out.

The supersymmetry theory as an extension of the Standard Model, predicts that one stable particle should be around today as a cosmological relic. Among these new particles, the neutralinos, could be the dark matter. If the dark matter halo of our Galaxy is made up of such particles, they will annihilate each other into fermion-antifermions pairs, and this will produce stable particles which could be detected as anti-protons, electrons and photons. Another way is to look for direct detection via laboratory experiments. Such new particles are scrutinized by direct and indirect searches since more than ten years. Our Galaxy is indeed a subject of intense activities because it could be a direct search for dark matter in studying astrophysical signatures.

Among all the signatures, our Galaxy presents three strong signatures in X-ray, $\gamma$-ray backgounds and a typical $e^{+} e^{-}$annihilation source in the Galactic Center. Among the 
other possibilities, the micro-lensing searches have been developed and used to probe the dark matter halo of our galaxy (see above). The possibility that the halo could be made of primordial black holes is not ruled out by observations. Recently, Seto and Cooray (2004) discussed also the future possibility of searching dark matter candidates using the spacebased gravitational wave detectors. They used this idea to test the possibility that the halo could be made of primordial black holes. According to Hawking predictions such primordial black holes will not suffer evaporation if their mass is greater than $10^{15} \mathrm{~g}$.

While the consequences of evaporating black holes has been widely analyzed in terms of particles production, the case for stable SMMBHs s has not been considered so far. In literature, the problem is analyzed generically and the mass range used are often very large from $10^{17} \mathrm{~g}$ to $10^{20} \mathrm{~g}$. We suggest the SMMBHs form the unseeing dark matter.

\section{Conclusions}

In this Paper, we show that there is a particular black hole mass which is very interesting for the dark matter problem. This mass of order of $10^{17} \mathrm{~g}$, corresponds to a typical Schwarzschild radius of order close to that of the proton. We explore this possibility in the light of existing galactic diffuse $\mathrm{X}$ and $\gamma$ backgrounds and with the strong source of annihilation lines at $511 \mathrm{keV}$ in the Galactic Center. Our approach is to find a natural explanation of such astrophysical backgrounds within an astrophysical dark matter scenario.

This also can provide an explanation for origin of the annihilation line appearance in the our Galaxy which has been detected in several balloon and space experiments. We combine $\mathrm{X}$-rays and $\gamma$-observations of the Galactic Center region to show that pair production can be a result of interactions of $\mathrm{X}$-rays and gamma photons emitted in this region. Pair production strongly depends on the compactness of the $\mathrm{X}$-rays and $\gamma$ emission region which is determined by $\mathrm{X}$-rays and $\gamma$ luminosity and size of the region.

For a velocity of the interstellar medium of much less than $10^{8} \mathrm{~cm} \mathrm{~s}^{-1}$ and the value of density column a few times $10^{22} \mathrm{~cm}^{-2}$, the optical depth for $511 \mathrm{keV}$ line is greater than one. We found for the observed X-rays and $\gamma$ luminosities and for the observed annihilation lines flux, the size of the X-ray and $\gamma$ emission area is $\sim 3 \times 10^{14} \mathrm{~cm}$. This size is consistent with the size of the area where the OB stars supply sufficient amount of fuel required for the $\mathrm{X}$-rays and $\gamma$ emissions from the Galactic Center region.

If we compute the total mass of SMMBHs inside the region of radius $R=10^{-4} \mathrm{pc}$, we obtain that this mass is about 70 solar masses or less (for this estimate see the end of section 5). One can then naturally ask if SMMBHs can be a candidate for dark matter 
as already proposed in literature ( e.g. Nemiroff \& Gould 1995, Marani et al. 1999). We compare this number to the dark matter mass computed using the singular power law cusp $\rho(r)=\rho_{0}\left(r / r_{0}\right)^{-\gamma}$ (where $0<\gamma_{\sim}^{<2}$ and $\rho_{0}=60-600 M_{\odot}$ is the dark matter density at radius $r_{0} \simeq 2 \mathrm{pc}$ ) often used in the dark matter model for the Galactic Center (see Moore et al. 1998 and Gnedin \& Primack 2004). It is evident that the value of the dark matter mass inferred from this model should be related to the radius of order a few parsecs and one should be careful to extrapolate this mass value to the radius of four orders of magnitudes less, namely to $R=10^{-4}$ pc. For this radius we find that the upper limit of the inferred mass using the dark matter model is at least of one order of magnitude less than that we obtain for SMMBHs.

Koyama (1996) and Muno et al. (2004a), Revnivtsev et al. (2004) noted that the Galactic Center exhibited intermittent activities with time-averaged energy generation rate $\sim 10^{41-42}$ ergs s$^{-1}$ comparable to Seyfert nuclei. Muno et al. (2004a) are left to conclude either that there is a significant shortcoming in understanding of mechanisms that heat the interstellar medium in Galactic Center region or that of a population of faint $\left(<10^{31}\right.$ ergs $\mathrm{s}^{-1}$ ) hard X-ray sources. Muno et al. (2004b) using a deep Chandra observations study to detail the origin of the point like sources near the Galactic Center. Possibly a single population of sources may dominate in this cluster. There are certainly many classes of objects present in this set of faint sources. We may suggest that some part of X-ray and $\gamma$-radiation of the Galactic Center, can originate in accreting flows into SMMBHs (that are really faint sources with their bolometric luminosity $<10^{22} \mathrm{ergs} \mathrm{s}^{-1}$ ) in which outer parts of flow emit X-rays and the innermost part of the flow emit $\gamma$-rays.

Finally we can conclude, that the only way to identify small mass black holes (SMMHHs) is by accretion mechanism in presence of a surrounding dense material. In the absence of this matter these SMMBHs are invisible and can be treated as dark matter, or invisible matter, i.e. without any observational appearance in the radiation. The crucial observational test of the SMMBHs as a model for a dark matter is detection of the blackbody radiation of $\sim 10$ $\mathrm{MeV}$ temperature from the Galactic Center region. In the energy range between $1-30 \mathrm{MeV}$ the photon energy flux of this emission should be of order $2 \times 10^{-2} \mathrm{~cm}^{-2} \mathrm{sr}^{-1} \mathrm{~s}^{-1} \mathrm{MeV}$ which is compatible with that which has already been detected by COMPTEL.

We appreciate productive discussion with Gerald Share and a help from Robert Duffin for editing of the early version of the manuscript. For this work, L.T. was partly supported by French Ministère de l'Education Nationale et de la Recherche with grant SSHN2004413077D. We thank the referee for his/her profound questions and we acknowledge the referee's contribution in the discussion section of this paper. 


\section{REFERENCES}

Aharonian, F., et al. 2004, A\&A, 495, L13

Armstrong, J.W., Rickett, B.J. \& Spangler, S.R. 1995, ApJ, 443, 209

Baganoff, F.K., 2003, ApJ, 591, 891

Boehm, C, Fayet, P., \& Silk, J. 2004, Phys. Rev, D69, 101302

Bykov, A.M. 2003, A\&A, 410, L5

Bykov, A.M. 2002, A\&A, 390, 327

Borozdin, K., Revnivtsev, M., Trudolyubov, S., Shrader, C, \& Titarchuk, L. 1999, ApJ, 517, 367 (BRT99)

Chakrabarti S.K. \& Titarchuk, L. G. 1995, ApJ, 455, 623

Chapuis, C.G.L. et al. 1991, Gamma-Ray Line Astrophysics, ed. P. Durouchoux \& N. Prantos (New York: AIP), 54

Cheng, L.X., 1997, ApJ, 481, L43

Chez, A.M., et al. 2004, ApJ, 601, L159

Chez, A.M., et al. 2003, ApJ, 586, L127

Churazov, E., Sunyaev, R., Sazonov, S. \& Varshalovich, D. 2005, MNRAS, 357, 1377

Dermer,C.D. \& Skibo, J.G. 1997, ApJ, 487, 57

Diehl, R., et al. 1995, A\&A, 298, 445

Dirac, P.A.M. 1930, Proc. Camb, Phil. Soc. 26, 361

Eisenhauer, F., et al. 2003, ApJ, 597, L121

Gehrels, N., et al. 1991, ApJ, 375, L13

Gnedin, O.Y., \& Primack, J.R. 2004, Phys. Rev. Lett. , 61, 302

Guessoum, N., Jean, P \& Gillard, W. 2005, A\&A, 436,171

Heitler, W. 1954, The Quantum Theory of Radiation, Oxford University Press, Oxford

Hunter, S.D. et al. 1997, ApJ, 481, 205 
Iyudin, A.F., et al. 1994, A\&A, 284, L1

Jean, P. 2003, A\&A, 407, L55

Jean, P. 2005, A\&A in press (astro-ph/0509298)

Kawai, N., et al. 1988, ApJ, 330, 130

Koyama, K., et al. 1996, PASJ, 48, 249

Kurfess; J.D., et al. 1992, ApJ, 399, L17

Laurent, P. \& Titarchuk, L. 1999, ApJ, 511, 289

Le Verrier, 1846, Astronomische Nachrichten, 25, 65

Leventhal, M. et al. 1993, ApJ, 405, L25

Leventhal, M., MacCallum, C.J., \& Stang, P.D. 1978, ApJ, 225, L11

Liu, S., Petrosian, V., \& Melia, F. 2004, ApJ, 611, L101

Liu, S., \& Melia, F. 2002, ApJ, 573, L23

Loeb, A. 2004, MNRAS, 350, 725

Longair, M.S. 1994, High Energy Astrophysics, Cambridge: University Press

Marani, G.F., Nemiroff, R.J., Norris, K. \& Bonnell, J.T. 1999, ApJ, 512, L13

Markevitch, M., Sunyaev, R.A., \& Pavlinsky, M. 1993, Nature 364, 40

Matz, S., et al. 1993, Nature 331, 416

Melia, F. \& Falcke, H. 2001, ARAA, 39, 309

Melrose, D.B. 1980, Plasma Astrophysics. Nonthermal Processes in Duffuse Magnetized Plasmas, New York: Gordon and Breach

Moore, B., et al. 1998, ApJ, 499, L5

Morris, M., \& Serabyn, E. 1996, ARA\&A, 34, 645

Muno, M., et al., 2005, ApJ, 622, L113

Muno, M., Baganoff, F.K, Bautz, M. W. et al. 2004a, ApJ, 613, 326 
Muno, M., Arabadjis, F. K, Baganoff, F.K, et al. 2004b, ApJ, 613, 1179

Oort J. H., 1932, Bull. Astron. Inst. Netherlands 6, 349

Narayan, R., et al. 1998, ApJ, 492, 554

Narayan, R. \& Yi, I. 1994, ApJ, 428, L13

Nemiroff, R.J. \& Gould, A. 1995, ApJ, 452, L111

Niel, M. et al. 1990, ApJ, 356, L21

Park, S. et al. 2004, ApJ, 603, 548

Parker, E. N. 1979, Cosmical Magnetic Fields, Clarendon Press, Oxford

Pavlinsky, M., Grebenev, S.A., \& Sunyaev, R.A., \& 1993, ApJ, 425, 110

Preddehl, P., \& Trumper,J. 1994, A\&A, 290, L29

Puls et al. 1996, A\&A, 305, 171

Purcell, W.R., et al. 1997, ApJ, 491, 725

Purcell, W.R., et al. 1993, ApJ, 413, L85

Ramaty, R., Skibo, J.G. \& Lingenfelter 1994, ApJS, 92, 393

Repolust, Puls \& Herrero 2004, A\&A, 415, 349

Revnivtsev, M.G., et al. 2004, A\&A, 425, L49

Schodel, R., et al. 2003, ApJ, 596, 1015

Schonfelder, V., et al. 2000, A\&ASS, 143, 145

Seto, N. \& A. Cooray, A. 2004, Phys. Rev. D, 70, 63512

Share, G.H., et al. 1990, ApJ, 358, L45

Shrader, C., \& Titarchuk, L.G. 2003, ApJ, 598, 168

Shrader, C., \& Titarchuk, L.G. 1999, ApJ, 521, L121 (ShT99)

Skinner, G.K., et al. 1987, Nature, 330, 544

Smith, D.M., Heindl, W.A. \& Swank, J.H. 2002, ApJ, 569, 362 
Strong, A.W., et al. 2006, A\&A in press (astro-ph/0509290)

Strong, A.W., Bloemen, H., Diehl, R., Hermsen, W., \& Schonfelder, V. 1998, Proc. 3rd. INTEGRAL Workshop (Eds. G. Palumbo, A.Bazano, \& C. Winkler) Astrophys. Lett. Comm. 39, 209

Strong, A.W. \& Moskalenko, I.V. \& Reimer,O.2004, ApJ, 613, 962

Strong, A.W. \& Moskalenko, I.V. \& Reimer,O.2000, ApJ, 537, 763

Strong, A.W. \& Moskalenko, I.V. 1999, Proc. 5th Compton Symposium (Eds. M.L. McConnell and J.M. Ryan) AIP Conference Proceedings, 510, 291

Sunyaev, R.A., Markevitch, M., \& Pavlinsky, M. 1993, ApJ, 407, 606

Sunyaev, R.A. \& Titarchuk, L.G. 1980, A\&A, 86, 121

Takeshima, T. et al. 1991, in proc. of 28th Yamada Conf., Frontiers of X-ray Astronomy, ed. Y. Tanaka, K. Koyama (Universal Academy Press, Tokyo), 421

Teegarden, B.J., et al. 1996, ApJ, 463, L75

Tanaka, Y., Inoue, H., \& Holt, S.S. 1995, PASJ, 46, L37

Titarchuk, L. \& Fiorito, R. 2004, ApJ, 612, 988

van Albada T. S., \& Sancisi R., 1986, Phil. Trans. R. Soc. Lond. A, 320, 447

Watson, M.G., Willingate, R., Grindlay, J.E., Hertz, P. 1981, ApJ, 250, 142

Wood, K.S., et al. 2001, ApJ, 563, 246

Yamauchi, S. et al. 1990, ApJ, 365, 532

Zwicky, F. 1937, ApJ, 86217 

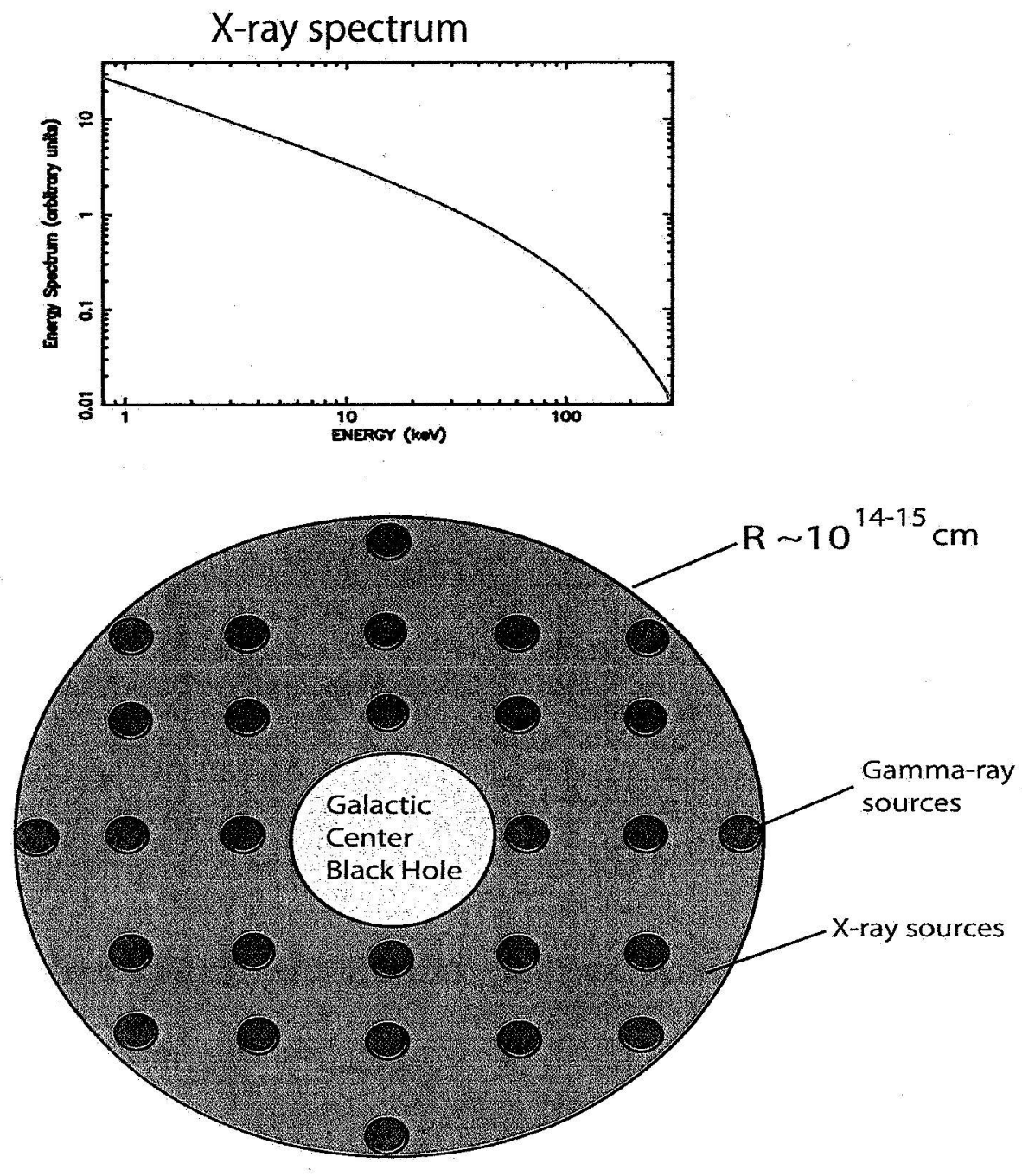

10 MeV Black Body Spectrum

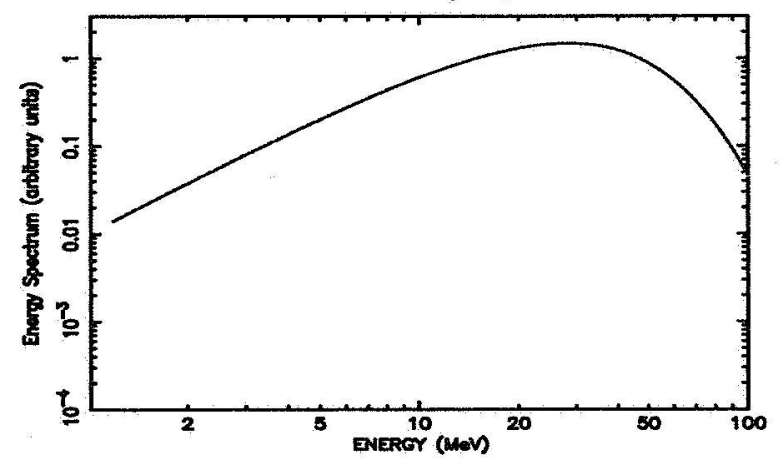

Fig. 1.- Cartoon picture of compact emission area near the Galactic Center. Sources of $\gamma$-ray region in blue, and sources of X-ray radiation in red. In upper and lower panels we show $\mathrm{X}$ and $\gamma$ rays spectra respectively. 